\title{
Repositioning of duloxetine to target pancreatic stellate cells
}

\author{
AKIKO SAGARA, KOHEI NAKATA, SOKICHI MATSUMOTO, WEIYU GUAN, TOMOHIKO SHINKAWA, \\ CHIKA IWAMOTO, NAOKI IKENAGA, KENOKI OHUCHIDA and MASAFUMI NAKAMURA
}

\author{
Department of Surgery and Oncology, Graduate School of Medical Sciences, \\ Kyushu University, Fukuoka 812-8582, Japan
}

Received May 19, 2021; Accepted July 29, 2021

DOI: 10.3892/ol.2021.13005

\begin{abstract}
Pancreatic cancer cells (PCCs) are surrounded by an abundant stroma, which is produced by pancreatic stellate cells (PSCs). PSCs promote tumor cell proliferation and invasion. The objective of the current study was to identify compounds that suppress PSC activation. Gene expression profiles of cancer-derived fibroblasts and normal fibroblasts were used, and the pathway analysis suggested altered pathways that were chosen for validation. It was found that the 'neuroactive ligand-receptor interaction' pathway from the Kyoto Encyclopedia of Genes and Genomes pathway analysis was one of the altered pathways. Several compounds related with this pathway were chosen, and changes in PSC activity were investigated using fluorescence staining of lipid droplets, reverse transcription-quantitative PCR, western blotting, and invasion and migration assays. Among these candidates, duloxetine, a serotonin-noradrenaline reuptake inhibitor, was found to suppress PSC activation and disrupt tumor-stromal interaction. Thus, duloxetine may be a potential drug for suppressing PSC activation and pancreatic cancer growth.
\end{abstract}

\footnotetext{
Correspondence to: $\mathrm{Dr}$ Kohei Nakata or Professor Masafumi Nakamura, Department of Surgery and Oncology, Graduate School of Medical Sciences, Kyushu University, 3-1-1 Maidashi, Higashi, Fukuoka 812-8582, Japan

E-mail:knakata@surg1.med.kyushu-u.ac.jp

E-mail:mnaka@surg1.med.kyushu-u.ac.jp
}

Abbreviations: $\alpha$ SMA, $\alpha$-smooth muscle actin; CAF, cancerassociated fibroblast; COL1, type-1 collagen; CTGF, connective tissue growth factor; DMEM, Dulbecco's modified Eagle's medium; ECM, extracellular matrix; FBS, fetal bovine serum; HGF, hepatocyte growth factor; IL-1 $\beta$, interleukin-1 $\beta$; IL-6, interleukin-6; KEGG, Kyoto Encyclopedia of Genes and Genomes; GPCR, G-protein coupled receptor; mRNA, messenger RNA; PBS, phosphate-buffered saline; PCCs, pancreatic cancer cells; PDAC, pancreatic ductal adenocarcinoma; POSTN, periostin; PSCs, pancreatic stellate cells; PP2A, protein phosphatase 2A; RT-qPCR, reverse transcription-quantitative polymerase chain reaction

Key words: pancreatic cancer, pancreatic stellate cells, drug repositioning, tumor microenvironment, duloxetine

\section{Introduction}

Pancreatic cancer is one of the most lethal cancers, with a 5 -year survival rate of $<9 \%$. While its incidence is increasing, its mortality rate has remained unchanged (1). Patient response to existing therapy is limited (2). Therefore, to overcome these issues, there is an increasing and urgent need to discover new treatment options.

In clinical practice, the traditional drug discovery process is expensive and takes over a decade (3). Thus, repositioning clinically evaluated drugs can be a strategy for the rapid detection and application of new alternative therapeutic approaches (4).

Pancreatic stellate cells (PSCs) are myofibroblast-like cells and are located in the pancreatic stroma (5). PSCs have the ability to produce components of the extracellular matrix (ECM) and make the stroma stiff. During pancreatic damage due to inflammation and metabolic stress, PSCs are activated by growth factors/cytokines released from neighboring cells (6). Activated PSCs support the proliferation of cancer cells, as shown by some studies assessing the relationship between cancer cells and PSCs (7). Therefore, inactivating PSCs could potentially be a novel approach for pancreatic cancer treatment.

The aim of this study was to determine a promising compound for inactivating PSCs. To achieve this, gene expression profiles of cancer-associated fibroblasts (CAF) and normal fibroblasts were analyzed, and neuroactive ligandreceptor interaction pathway related to the cancer-associated PSCs was identified. We focused on duloxetine, a serotoninnoradrenaline reuptake inhibitor, as a potential compound for suppressing the activity of PSCs.

\section{Materials and methods}

Cells and culture conditions. SUIT-2 [Japan Health Science Research Resources Bank (JCRB), Osaka, Japan] was purchased and maintained in Dulbecco's modified Eagle's medium (DMEM) (Sigma-Aldrich Co.), supplemented with $10 \%$ fetal bovine serum (FBS). Human PSCs were generated in our laboratory from two fresh surgical specimens of pancreatic ductal adenocarcinoma (PDAC), using the outgrowth method (8), and were maintained in DMEM with $10 \%$ FBS. Human Pancreatic Stellate Cells (HPaSteC cells, cat. no. 3830; ScienCell Research Laboratories) were maintained in Stellate 
Cell Medium (cat. no. 5301; ScienCell Research Laboratories), according to the manufacturer's instructions. All cells were free of mycoplasma contamination. The cells were authenticated using short tandem repeat (STR) profiling. Cells at passages 3-8 were used for assays.

Human PDAC organoid were established as described previously $(9,10)$. A PDAC sample from a 75 -year-old male who underwent pancreatoduodenectomy in 2015 at Kyushu University was collected. Surgical specimens of PDAC were minced and digested with a Tumor Dissociation Kit (human, cat. no. 130-095-929; Miltenyi Biotec). Then, the tissue was embedded in growth factor-reduced (GFR) Matrigel (cat. no. 356231; BD Bioscience) and cultured in a human complete medium at $37^{\circ} \mathrm{C}$ for 14 days. The human complete medium used was Advanced DMEM/F12 (cat. no. 12634-010; Invitrogen), supplemented with 1M HEPES (Invitrogen), GlutaMax (cat. no. 35050-061; Invitrogen), penicillin/streptomycin (cat. no. 15140122; Invitrogen), B27 (cat. no. 17504044; Invitrogen), N-acetyl-L-cysteine (cat. no. A9165; Sigma-Aldrich Co.), Wnt-3a (cat. no. 5036-WN-010; R\&D Systems), R-Spondin 1 (cat.no. 120-38; Peprotech),Noggin (cat.no.120-10C; Invitrogen), epidermal growth factor (EGF, cat. no. AF-100-15; Peprotech), fibroblast growth factor (FGF, cat. no. 100-26; Peprotech), Nicotinamide (cat. no. N0636; Sigma-Aldrich Co.), Y-27263 (cat. no. Y0503; Sigma-Aldrich Co.), and A83-01 (cat. no. 2939/10; R\&D Systems). Organoid area measurements were performed using KEYENCE BZ-X analyzer.

Identification of differentially expressed genes from public microarray data. Public gene expression profiles for GSE53524 were obtained from Gene Expression Omnibus (GEO, http://www.ncbi.nlm.nih.gov/geo). Data was analyzed, and a set of genes differentially expressed in CAFs and normal fibroblasts were identified. P-values $<0.05$ for microarray data were selected. Pathway enrichment analysis of targets was performed using the DAVID v6.8 (https://david.ncifcrf.gov/). The Kyoto Encyclopedia of Genes and Genomes (KEGG) pathway enrichment analysis was performed for identified up- and down-regulated genes. $\mathrm{P}<0.05$ was considered to indicate statistical significance.

Drugs. Duloxetine hydrochloride (cat. no. 040-34071, Wako Pure Chemicals) was dissolved in dimethyl sulfoxide (DMSO) at a final concentration of $10 \mathrm{mM}$ and stored at $-20^{\circ} \mathrm{C}$. Paroxetine hydrochloride (cat. no. 168-24431, Wako Pure Chemicals), desloratadine (cat. no. D3787, TCI Chemicals), promethazine, amitriptyline hydrochloride (cat. no. 013-12882, Wako Pure Chemicals), and chlorpromazine hydrochloride (cat. no. 033-10581, Wako Pure Chemicals) were dissolved in DMSO at a concentration of $10 \mathrm{mM}$ and stored at $-20^{\circ} \mathrm{C}$.

Lipid accumulation assay. BODIPY ${ }^{\circledR}$ staining was used for fixed cells as described previously (11). PSCs were seeded on glass-bottomed multi-well plates (P06G-1.5-14-F, MatTek Corporation) at $1 \times 10^{5}$ cells/well. After $24 \mathrm{~h}$ of incubation, the medium was aspirated and fresh DMEM containing 10\% FBS was added. For the drug treatment group, each drug was dissolved in the same DMEM at a concentration of $10 \mu \mathrm{M}$. We have previously reported that chloroquine inhibits PSC activation (11). We conducted a drug screening for PSCs to select other candidate drugs that inhibit PSC activation (12). Chloroquine had been shown to inhibit PSC activity at a concentration of $10 \mu \mathrm{M}$, and since this concentration was used as the standard for our previous screening, we used this concentration as well. After $48 \mathrm{~h}$ of incubation, cells were washed with PBS and fixed with $4 \%$ paraformaldehyde phosphate buffer solution and stained with $1 \mathrm{mg} / \mathrm{ml}$ 4,4-Difluoro-1,3,5,7,8-Pentamethyl4-Bora-3a,4a-Diaza-s-Indacene (BODIPY ${ }^{\circledR}$ 493/503, cat. no. D-3922; Life Technologies) and Hoechst 33342 solution (1 $\mathrm{mg} / \mathrm{ml}$, cat. no. H342; Dojindo) for $30 \mathrm{~min}$ in the dark and at room temperature. Cells were washed with phosphatebuffered saline, and images were captured using a fluorescent microscope.

For live cells, lipid accumulation was stained by Lipidye ${ }^{\circledR}$ according to the manufacturer's instructions. Cells were seeded in glass-bottomed multi-well plates at a density of $1 \times 10^{5}$ cells/well. After $24 \mathrm{~h}$ of incubation, the medium was aspirated, and a fresh medium with the indicated concentrations of drugs or DMSO was added. After culturing for each period, cells were stained with $1 \mathrm{mg} / \mathrm{ml}$ 1,3-Diphenyl-2-[4-(N,N-diphenylamino)phenyl] benzo[b] phosphole-P-oxide (Lipidye ${ }^{\circledR}, 405 / 520$ ) and $1 \mathrm{mg} / \mathrm{ml}$ Hoechst 33342 for $2 \mathrm{~h}$ in the dark at $37^{\circ} \mathrm{C}$. Images were captured using a fluorescence microscope.

Cell viability assay. Cell Titer-Glo Luminescent Cell Viability Assay (Promega Corp.) was used for assaying. PSCs $\left(1 \times 10^{3}\right.$ cells/well) were seeded in 96-well polystyrene cell culture microplates (cat. no. 655083, Greiner Bio-One International) and incubated at $37^{\circ} \mathrm{C}$ in a humidified atmosphere with $10 \% \mathrm{CO}_{2}$. After $24 \mathrm{~h}$, the medium was aspirated and fresh DMEM containing 10\% FBS and the candidate drugs at each concentration were added. Fluorescence was measured after $48 \mathrm{~h}$. The emission value was measured as a control using a microplate reader (Infinite F200; Tecan), according to the manufacturer's instructions. Cell viability was evaluated as the ratio to the control.

Reverse transcription-quantitative polymerase chain reaction. For duloxetine treatment, we used the concentration of $10 \mu \mathrm{M}$. Total RNA was extracted from PSCs using a High Pure RNA Isolation kit (cat. no. 11828665001, Roche), according to the manufacturer's instructions. Reverse transcription-quantitative polymerase chain reaction (RT-qPCR) was performed using STBR Green RT-PCR kit (cat. no. 170-8892; Bio-Rad Laboratories) and the CFX96 Real-Time PCR System (Bio-Rad Laboratories). Primers were purchased from Takara (Shiga, Japan). The primer sequences are listed in Table SI. The reactions were incubated at $50^{\circ} \mathrm{C}$ for $10 \mathrm{~min}, 95^{\circ} \mathrm{C}$ for $1 \mathrm{~min}, 95^{\circ} \mathrm{C}$ for $10 \mathrm{sec}, 60^{\circ} \mathrm{C}$ for $30 \mathrm{sec}$ for 39 cycles, $60^{\circ} \mathrm{C}$ for $5 \mathrm{sec}$, and $95^{\circ} \mathrm{C}$ for $5 \mathrm{sec}$. The quantity of messenger RNA (mRNA) was calculated using the $2^{-\Delta \Delta C q}$ method (13). mRNA expression was normalized using glyceraldehyde-3-phosphate dehydrogenase.

Western blotting. Cells were incubated for $48 \mathrm{~h}$ with each concentration of duloxetine $(0,1,5$ and $10 \mu \mathrm{M})$. Proteins were extracted using PRO-PREP Protein Extraction Solution (cat. no. 17081, iNtRON Biotechnology), according to the manufacturer's instructions. The protein concentration was measured using a NanoDrop 1000 Spectrophotometer 
(version 3.8.1; Thermo Fisher Scientific). After $48 \mathrm{~h}$ of incubation, cells were washed, and the medium was changed to DMEM with $0 \%$ FBS and was cultured overnight. Culture supernatants were collected in a centrifuge tube (cat. no. UFC900324, AmiconUltra; Merck Millipore) and centrifuged at 7,500 rpm for $30 \mathrm{~min}$. Halt protease and phosphatase inhibitors (cat. no. 78442; Thermo Fisher Scientific) were added at a 1:1,000 dilution into the concentrated supernatants. Collected proteins $(20 \mu \mathrm{g}$ per lane from whole cell lysate) were fractionated on 4-15\% Mini-PROTEAN TGX Precast Gels (cat. no. 456-1084; Bio-Rad Laboratories) and transferred to Trans-Blot Turbo Mini PVDF Transfer Packs (cat. no. 170-4156, Bio-Rad Laboratories) using a Trans-Blot Turbo Transfer Starter System (Bio-Rad Laboratories). The membrane was incubated at $4^{\circ} \mathrm{C}$ overnight with anti-Akt (Rabbit mAb, 1:1,000, cat. no. 4691; Cell Signaling Technology), anti-pAkt (Rabbit mAb, 1:1,000, cat. no. 4060; Cell Signaling Technology), anti- $\alpha$ SMA (Mouse mAb, 1:2,000, cat. no. M0851; Dako), anti- $\alpha$ tubulin (Rabbit pAb, 1:2,000, cat. no. bs-50500R; Bioss), anti-Erk (Rabbit mAb, 1:1,000, cat. no. 4695; Cell Signaling Technology), anti-pErk (RabbitmAb,1:1,000,cat.no.4370S; CellSignaling Technology), anti-fibronectin (Rabbit pAb, 1:1,000, cat. no. ab2413; Abcam), anti-COL1A1 (Rabbit pAb, 1:1,000, cat. no. 84336S; Cell Signaling Technology), anti-IL-6 (Mouse mAb, 1:1,000, ab9324; Abcam), anti-periostin (POSTN; Rabbit pAb, 1:1,000, cat. no. ab14041; Abcam), anti-PP2A (Rabbit mAb, 1:1,000, cat. no. ab32104; Abcam) or mouse anti- $\beta$-actin (Mouse mAb, 1:10,000, cat. no. 81178; Santa Cruz Biotechnology) antibodies. Membranes were then probed with horseradish peroxidaseconjugated secondary antibodies (Santa Cruz Biotechnology). Immunoblots were detected by enhanced chemiluminescence using the ChemiDoc XRS System (Bio-Rad Laboratories) and were analyzed using Quantity One Software (Bio-Rad Laboratories). Each experiment was repeated three times.

Matrigel invasion and migration assays. Cell invasion and migration were measured by counting the number of cells through Transwell chambers with $8-\mu$ m pores (cat. no. 353097; BD Biosciences). For invasion assays, Transwell inserts were coated with $20 \mu \mathrm{g} /$ well Matrigel (cat. no. 354234; Corning). After confirming the half-maximal inhibitory concentration for PSCs, we used $10 \mu \mathrm{M}$ for invasion and migration assay, because this concentration did not exceed the $\mathrm{IC}_{50}$ for PSCs. The procedure involved: a) control of DMEM with 10\% FBS, b) supernatant collected from PSC, c) DMEM with $10 \% \mathrm{FBS}$ containing duloxetine at $10 \mu \mathrm{M}$, and d) supernatant collected from PSC cultured with $10 \mu \mathrm{M}$ duloxetine added in the 24-well culture plate (cat. no. 353504; Corning). SUIT-2 cells $\left(4 \times 10^{4}\right)$ in $250 \mu \mathrm{l}$ DMEM containing $2 \%$ FBS were seeded in the upper chamber and placed in a 24 -well culture plate. After $48 \mathrm{~h}$ of incubation for invasion assays or $24 \mathrm{~h}$ for migration assays, upper chambers were fixed with $70 \%$ ethanol. Cells were washed, stained with hematoxylin and eosin, and counted in five random fields at x100 magnification using a BZ-X Analyzer (Keyence). Samples were evaluated in a blinded manner. The experiments were repeated three times.

Statistical analysis. All values are expressed as mean \pm standard deviation $(S D)$. For comparison between two groups only, data was analyzed with an unpaired two-tailed t-test. For comparison of multiple groups, comparisons were conducted using ANOVA, followed by Tukey-Kramer method. A P-value $<0.05$ was considered to indicate a statistically significant difference.. Experiments were repeated at least three times. All statistical analyses were performed using GraphPad Prism 8.

\section{Results}

Gene expression profiles of cancer-associated PSCs. To identify the potential therapeutic targets of cancer-associated PSCs, we examined the gene expression profiles from public microarray data obtained from murine pancreatic CAFs and normal fibroblasts (GSE53524) (14). To identify the genes associated with CAFs in comparison to normal fibroblasts, probe sets with a P-value $<0.05$ for microarray data were selected. We found that many up-regulated genes were associated with several pathways of the KEGG pathway enrichment analysis (Fig. 1A). The hippo-signaling pathway and calcium-signaling pathway are reported potential target pathways in PSC activation $(15,16)$. On the other hand, neuroactive ligand-receptor signaling in PSCs has not been studied. Therefore, we focused on this pathway.

To select candidate compounds, we referred to previous studies and focused on the gene targets in this pathway and selected compounds related to the pathway (17). In addition, we previously demonstrated high-throughput drug screening for detecting compounds that suppress the activity of PSCs (12). The result from this screening showed that these six compounds were included as candidate drugs for inactivating PSC function. Therefore, we selected these six compounds and confirmed their role in PSC inactivation. The following drugs were selected and evaluated: duloxetine, a 5-hydroxytryptamine and noradrenaline reuptake inhibitor; paroxetine, a selective serotonin (5-HT) reuptake inhibitor; desloratadine and promethazine, first-generation antihistamines; amitriptyline, a tricyclic antidepressant; and chlorpromazine, a dopamine receptor antagonist. These compounds are G-protein coupled receptor (GPCR) inhibitors, and they showed an affinity to GPCR (Table SII). Next, to assess the effect of these candidates on the PSC state, lipid droplet accumulation assaying for detecting quiescent PSCs was performed, as previously reported (11). Lipid accumulation in the cytoplasm was observed following treatment with five out of six compounds, and duloxetine showed the highest accumulation of lipid droplets (Fig. 1B). Therefore, duloxetine (Fig. 1C) was selected as a potential compound for suppressing the activity of PSCs.

Duloxetine suppressed the activity of PSCs. These results led us to further investigate the effects of duloxetine on PSC activation. First, we investigated the half-maximal inhibitory concentration of duloxetine for patient-derived PSCs and HPaSteC cells (hPSC). These concentrations ranged from 19.2 to $25.2 \mu \mathrm{M}$ (Fig. 2A). Next, we investigated whether duloxetine suppresses the PSC activity, according to the previous report $(6,11)$. The quiescent state is a reversible state (18). Therefore, we used live cells and demonstrated the changes of lipid droplets in the cytoplasm between duloxetine 


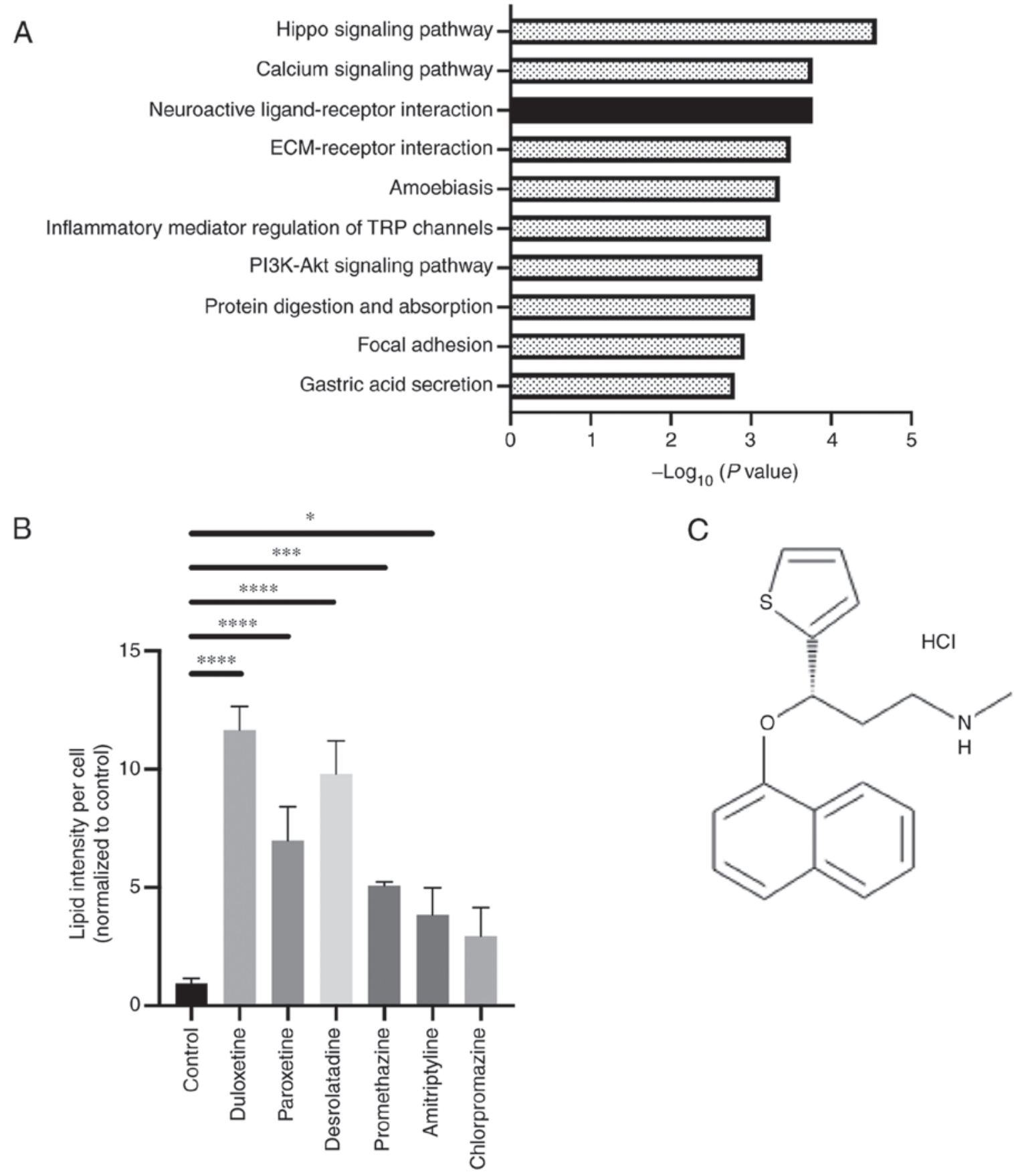

Figure 1. Discovery of a potential pathway for the identification of novel compounds for pancreatic stellate cells via pathway analysis. (A) Kyoto Encyclopedia of Genes and Genomes pathway analysis of cancer-associated fibroblasts vs. normal fibroblasts from the public database. The top 10 altered pathways are ranked according to their P-values. (B) Lipid accumulation was calculated as the resultant fluorescence intensity after treatment with drugs (10 $\mu \mathrm{M}$ each) related to the 'neuroactive ligand-receptor interaction' pathway. The control group was treated with $0.025 \%$ dimethyl sulfoxide. (C) Structure of duloxetine. ${ }^{*} \mathrm{P}<0.05,{ }^{* * *} \mathrm{P}<0.001,{ }^{* * * *} \mathrm{P}<0.0001$ vs. control group.

treatments. To determine the changes in lipid droplets in live PSCs, Lipidye ${ }^{\circledR}$ staining was used for immunofluorescent staining (19). Lipid accumulation was observed with duloxetine treatment. Further, after washout and replacement of the drug-free medium, the intracellular lipid droplets disappeared (Fig. 2B). Next, we determined the expression of the PSC activation markers, alpha-smooth muscle actin ( $\alpha$ SMA), and ECM proteins (collagen I, fibronectin and POSTN) by RT-qPCR and western blotting of whole-cell lysates. Duloxetine reduced the protein and mRNA expression of these markers in PSCs
(Fig. 2C and D), suggesting that duloxetine suppressed the activity of PSCs. This effect was reversible, suggesting that duloxetine induced PSC quiescence.

Duloxetine suppressed the proliferation of patient-derived organoids. Next, to determine the effects of duloxetine on cancer cell growth, we used a PCC 2D culture and proliferation of human PCC cell line was directly attenuated by duloxetine treatment (Fig. 3A). It has been widely suggested that organoid models can epitomize in vivo-like growth and 

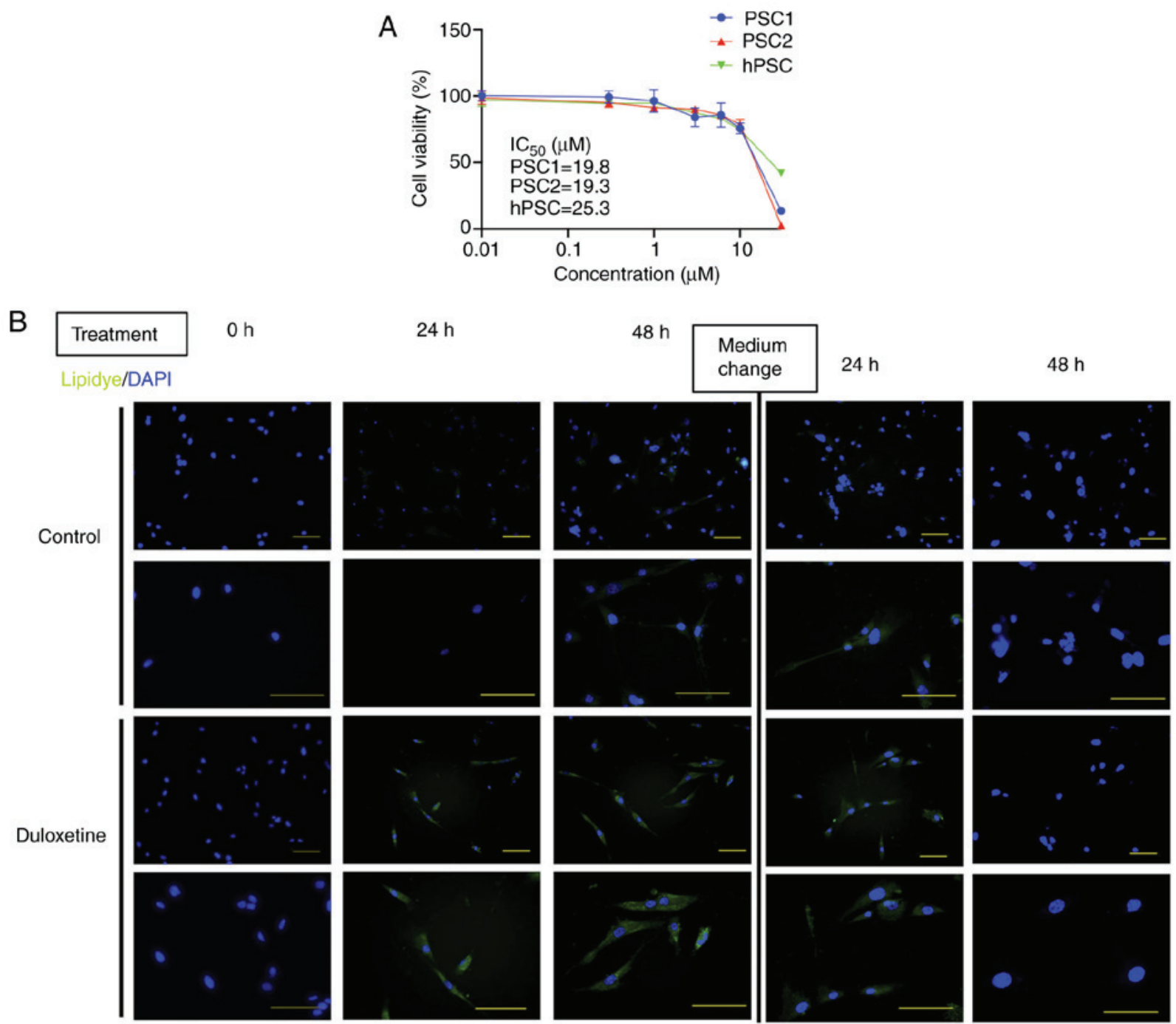

C
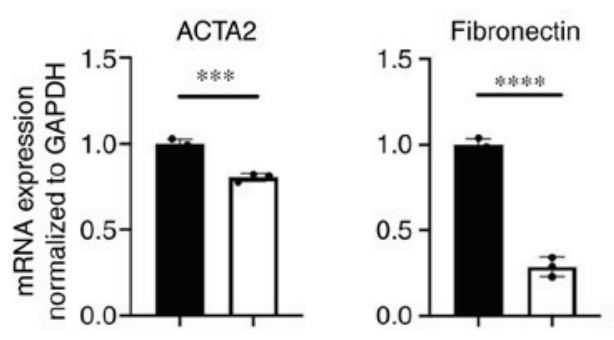

D
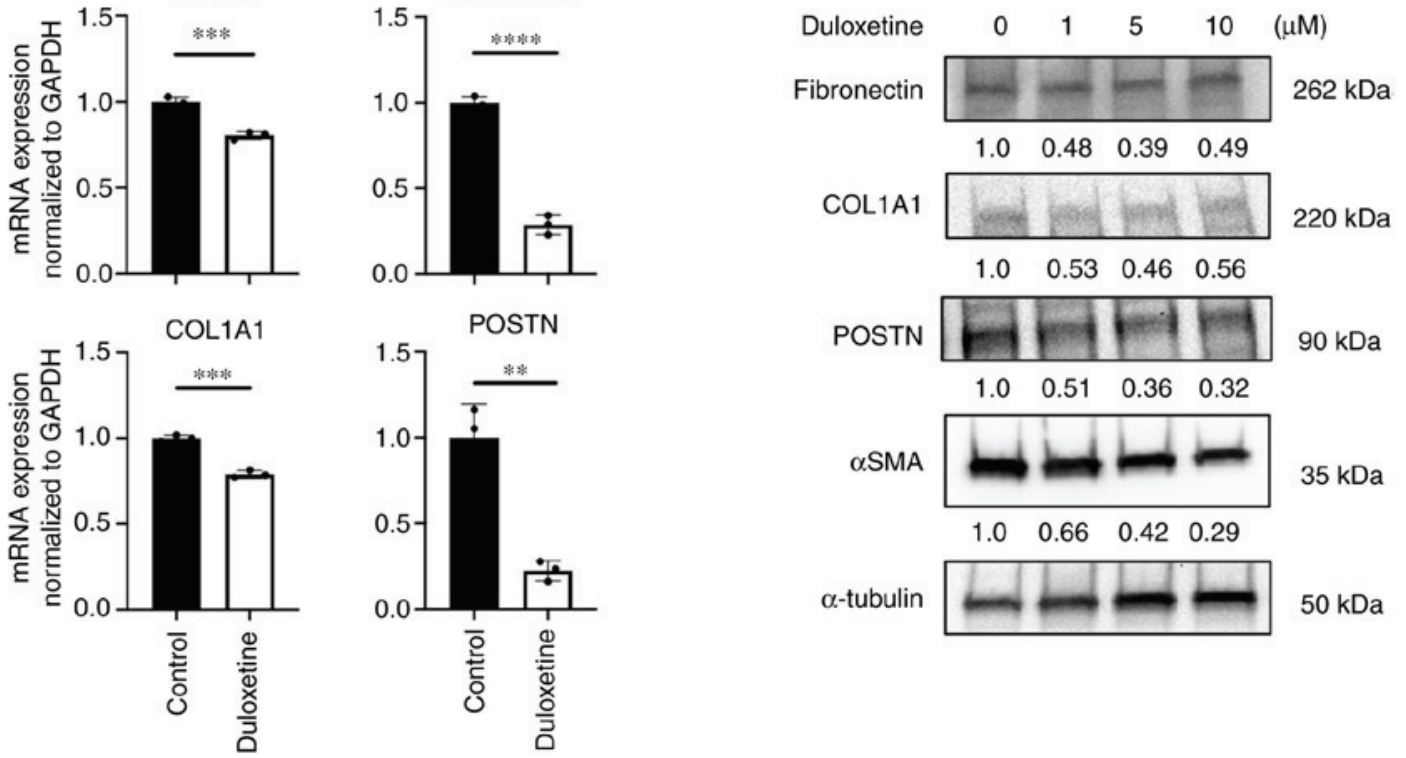

Figure 2. Duloxetine induces PSCs into a quiescent state. (A) Cell viability of PSCs in vitro. Each $\mathrm{IC}_{50}$ value is presented in the graph. (B) Representative photomicrographs of LipiDye staining of PSCs taken 0,24 and $48 \mathrm{~h}$ after duloxetine treatment. DAPI was used for nuclear staining. After $48 \mathrm{~h}$, the medium was washed off, fresh Dulbecco's modified Eagle's medium with 10\% FBS was added, and the culture was continued. Magnifications, x200 (upper panels) and x400 (lower panels). Scale bar, $100 \mu \mathrm{m}$. (C) mRNA expression levels of $\alpha$ SMA and ECM proteins in PSCs. mRNA expression levels were normalized to GAPDH expression and are presented as the fold-change in gene expression relative to control PSCs. (D) Western blotting of $\alpha$ SMA and ECM proteins from whole cell lysate of PSCs. Values indicate densitometric ratios normalized to $\alpha$-tubulin. ${ }^{* *} \mathrm{P}<0.01,{ }^{* * * *} \mathrm{P}<0.001,{ }^{* * * * *} \mathrm{P}<0.0001$ vs. control group. PSCs, pancreatic stellate cells; $\alpha$ SMA, $\alpha$-smooth muscle actin; ACTA2, actin $\alpha 2$ smooth muscle; ECM, extracellular matrix; GAPDH, glyceraldehyde-3-phosphate dehydrogenase; hPSC, human pancreatic stellate cells; COL1A1, $\alpha-1$ type-1 collagen; POSTN, periostin. 
A

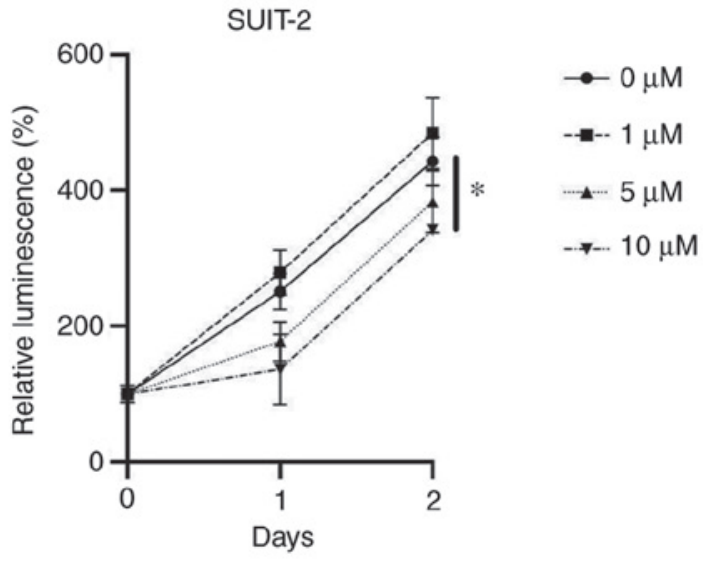

B

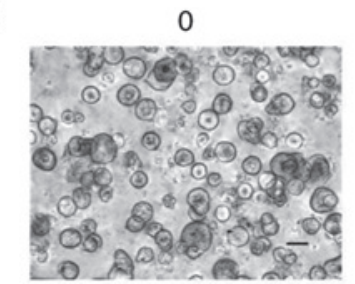

15
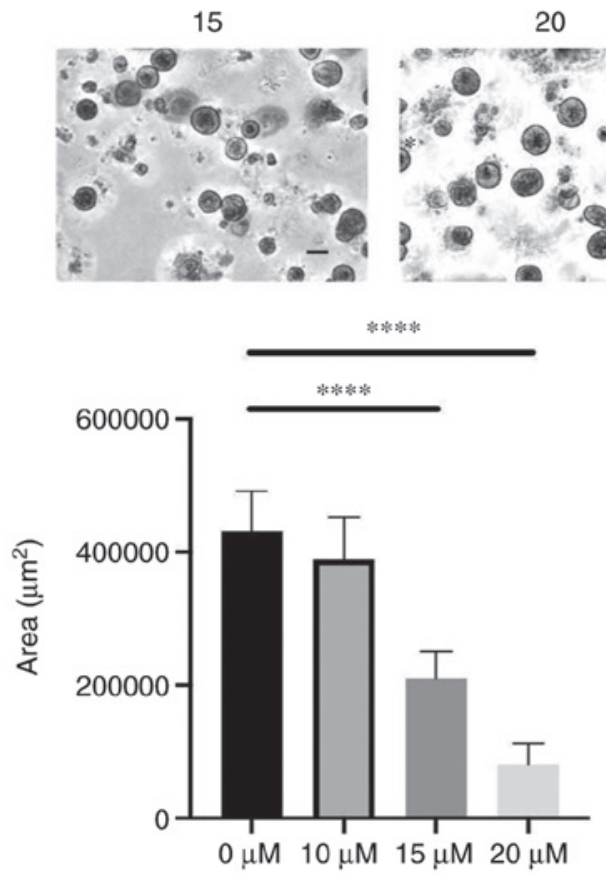

Figure 3. Duloxetine suppresses pancreatic cancer cell line and organoid growth. (A) Proliferation assay for SUIT-2 cells in vitro. (B) Representative photomicrograph of an organoid derived from a human surgical specimen. Each organoid was treated with duloxetine at various concentrations $(10,15$ and $20 \mu \mathrm{M})$. Graph shows the quantification of grown organoids in five random fields at x100 magnification. Scale bar, $100 \mu \mathrm{m} .{ }^{*} \mathrm{P}<0.05,{ }^{* * * * *} \mathrm{P}<0.0001$ vs. $0 \mu \mathrm{M}$ group.

differentiation of tissues as compared to 2D cultures (20). We found that duloxetine suppressed organoid formation and growth in organoids developed from the human surgical specimens (Fig. 3B), suggesting that duloxetine inhibits the growth of tumor organoids.

Duloxetine suppressed tumor-stromal interaction. Previous data showed that activated PSCs affect pancreatic cancer cell invasion and migration (21). To confirm whether duloxetine suppresses tumor-stromal interaction between pancreatic cancer cells (PCCs) and PSCs, an invasion and migration assay was performed. Compared to the control group, duloxetine itself did not attenuate PCC invasion and migration (Fig. 4A). Consistent with previous reports, co-culture with PSC supernatant stimulated PCC invasion and migration. However, this enhancement effect was abolished by co-culturing with a duloxetine-treated PSC supernatant (Fig. 4A and B), supporting the hypothesis that duloxetine suppresses the activity of PSCs and production of growth factors and cytokines that promote tumor-stromal interaction.

To further confirm the mechanism of duloxetine action, we used western blotting to determine the major secreted proteins and cytokines that attenuate tumor-stromal interaction from the PSC supernatant and whole cell lysates. Growth factors and pro-inflammatory cytokines released from adjacent cells cause PSC activation (22). Activated PSCs maintain their activated state by autocrine stimulation of cytokines, leading to proliferation, migration, and overproduction of the ECM (23). The duloxetine-treated PSC supernatant had fewer ECM protein amounts than those in the control (Fig. 4C). mRNA expression of connective tissue growth factor (CTGF), hepatocyte growth factor (HGF), interleukin-1 $\beta$ (IL-1 $\beta$ ), and interleukin-6 (IL-6) in PSCs decreased significantly with duloxetine treatment (Fig. 4D). These findings suggested that duloxetine suppressed PSC activation and reduced the quantity of the proteins and mRNA expression that contribute to stromal stiffness and tumor-stromal interactions.

Duloxetine suppressed the activation of Akt-ERK pathway via PP2A activation. Protein phosphatase 2A (PP2A) is known as a tumor suppressor and is involved in the regulation of many cellular functions, including extracellular signal-regulated kinase (ERK) signaling (24). A previous study revealed that PP2A contributes to cellular quiescence $(24,25)$. To confirm that duloxetine affects PP2A and suppresses PSC activation, the protein expression was confirmed. In the western blot analysis, the PP2A expression level was increased by duloxetine treatment (Fig. 4E). Also, duloxetine treatment decreased in Akt and ERK phosphorylation (Fig. 4E). These data suggested that duloxetine reversed PP2A activation, which led PSCs into a quiescent state.

\section{Discussion}

In this study, the pathway analysis using a public microarray data of CAFs showed aberration hubs in the "neuroactive ligand-receptor interaction' pathway. This led to the identification of a promising compound, duloxetine. Duloxetine suppressed PSC activation and disrupted tumor-stromal interaction. Together, these experiments elucidated the potential role of duloxetine as an alternative drug for pancreatic cancer treatment.

From the microarray analysis, other pathways identified in the top 10 list were previously reported as targets of cell proliferation and adhesion. We focused on the neuroactive ligand-receptor interaction pathway because it is the only pathway whose relationship with PSC activation has not been reported. Lipid accumulation was detected in cells treated with all compounds related to this pathway; 
A

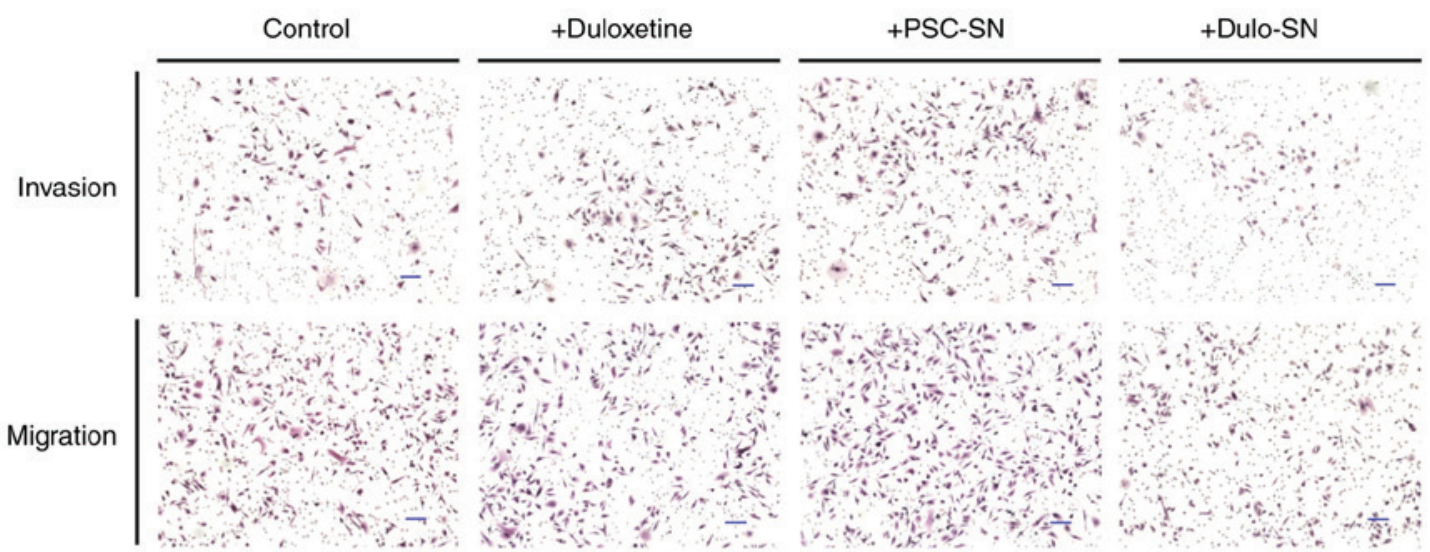

B

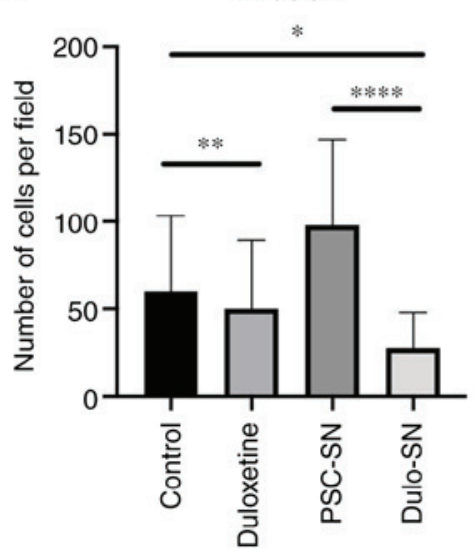

Migration

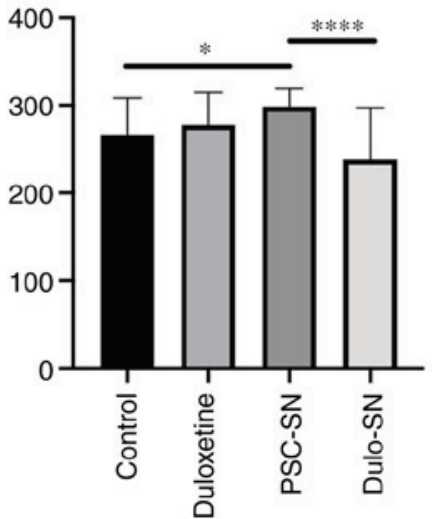

C

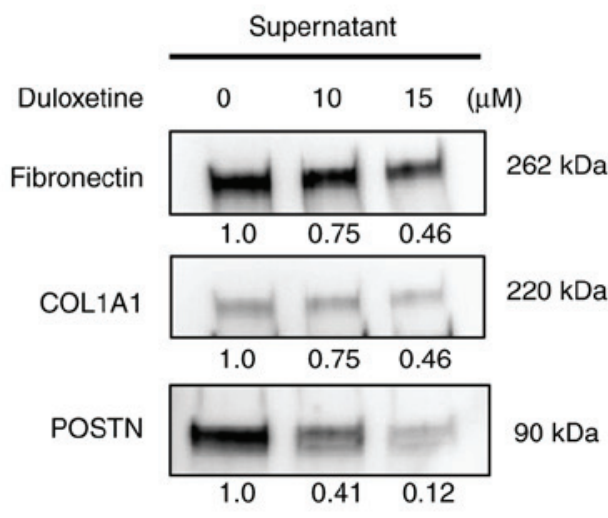

D
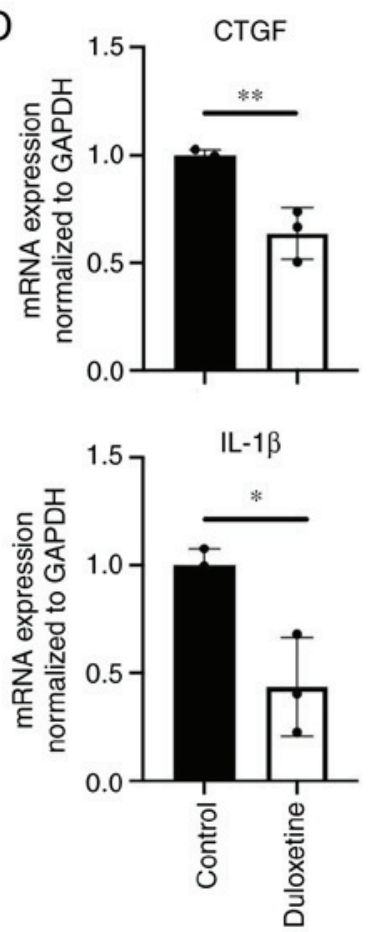
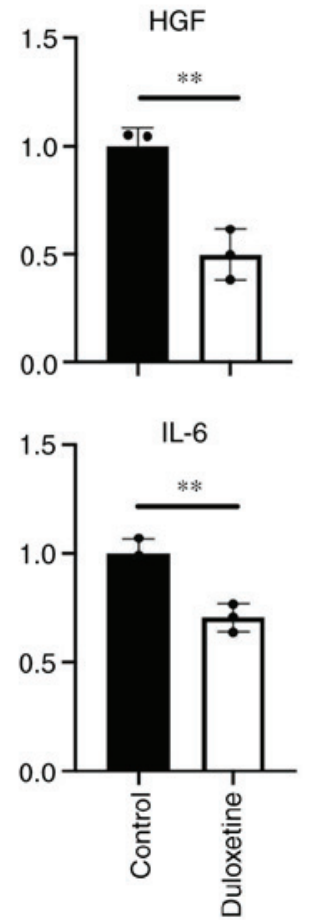

E

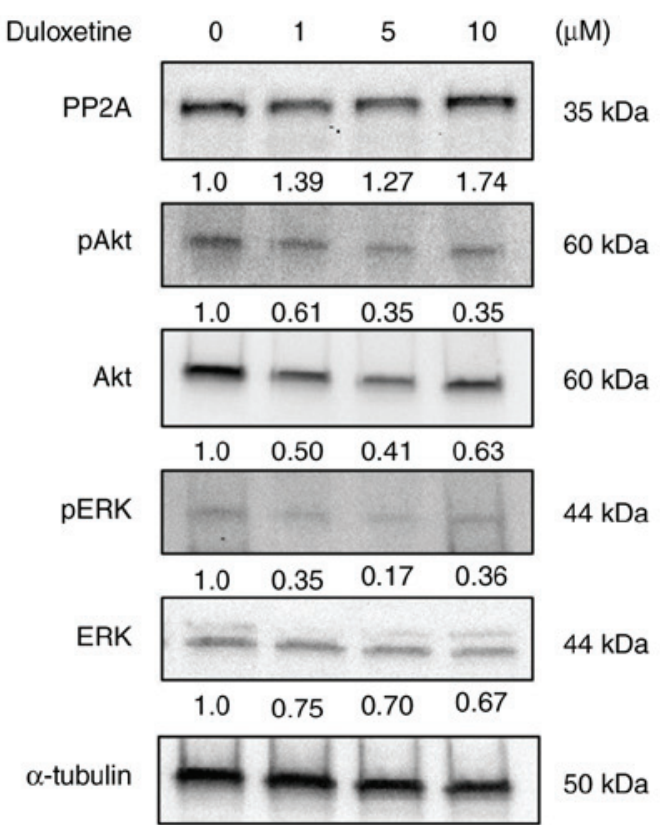

Figure 4. Duloxetine suppresses tumor-stromal interactions by attenuating secretomes from PSCs. (A) Representative photomicrographs of invading and migrating SUIT-2 cells in monoculture and indirect coculture with drugs or supernatants following hematoxylin and eosin staining. Magnification, x100. Scale bar, $100 \mu \mathrm{m}$. (B) Graphs show the number of invading and migrating SUIT-2 cells. (C) Western blotting of extracellular matrix proteins in PSC supernatant and drug-treated PSC supernatant. (D) Relative mRNA expression of growth factors and cytokines associated with tumor-stromal interactions in PSCs treated with duloxetine. The expression levels of each gene were normalized to GAPDH. (E) Western blotting of PP2A and related proteins in whole cell lysates of PSCs treated with duloxetine at various concentrations $(1,5$ and $10 \mu \mathrm{M})$. Values indicate densitometric ratios normalized to $\alpha$-tubulin. ${ }^{*} \mathrm{P}<0.05,{ }^{* *} \mathrm{P}<0.01$, ${ }^{* * * * *} \mathrm{P}<0.0001$ vs. control group. PSCs, pancreatic stellate cells; PSN-SN, PSC supernatant; Dulo-SN, supernatant from PSCs treated with duloxetine; PP2A, protein phosphatase 2A; COL1A1, $\alpha-1$ type-1 collagen; POSTN, periostin; CTGF, connective tissue growth factor; HGF, hepatocyte growth factor; IL-1 $\beta$, interleukin-1 $\beta$; IL-6, interleukin-6; p, phosphorylated. 
therefore, we selected duloxetine, which showed the highest lipid accumulation. Most of the drugs' targets related to this pathway are G protein-coupled receptors (GPCRs). GPCRs are located on the cell surface and are related to tumor cell proliferation and invasion in various types of tumors $(26,27)$. More than $30 \%$ of approved drugs exert their therapeutic effects by acting on GPCRs (28). Therefore, they are recognized as potential targets of anti-cancer drugs (29). There are some reports that show the relationship between PSC activation and GPCRs. Cortes et al observed that G protein-coupled estrogen receptor, a GPER, is a mechanosensor of PSCs and can remodel stromal tissue, thereby preventing tissue stiffness (30). Other research indicated that GPR68, a proton-sensing GPCR, was expressed in CAFs and acted as a mediator of the tumor microenvironment (31). These prior studies support targeting one of the GPCRs as a promising approach for suppressing PSC activation. However, this study did not clarify whether the suppression of activation is mediated by GPCRs and what structural differences exist in the GPCRs that exert therapeutic effects. Given that GPCRs are widely expressed across tissues and their role is mediated through various signaling pathways (32), further experiments are required to elucidate the mechanism of GPCR in PSC function.

The PSC supernatant enhanced the invasion and migration of PCCs as previously reported. These findings are due to enhanced tumor-stromal interactions (22). PCC invasion and migration were interrupted to a further extent by co-culturing with the supernatant from duloxetine-treated PSCs and not with duloxetine itself. This suggested that cytokines or proteins secreted by PSCs were suppressed by duloxetine. During activation, PSCs receive various stimuli from adjacent cells, and activated PSCs are potent to secrete inflammatory cytokines and ECM proteins (33). We calculated ECM proteins and cytokines secreted by activated PSCs and observed that these secretions were decreased by duloxetine. Together, we concluded that duloxetine not only has a toxic effect on PSCs, but can induce inactive PSC.

Treatment strategies for reshaping the tumor stroma have been widely discussed and investigated in the past decades. In clinical settings, expression of pro-tumorigenic markers and ECM components is correlated with a worse prognosis (34). In this study, PSCs treated with duloxetine produced less ECM components, including collagen and POSTN that constitute the tumor-supportive microenvironment (35). Therefore, converting pro-tumor PSCs into tumor-suppressing cells may be an ideal approach for treating pancreatic tumors. It has also been revealed that different fibroblast subtypes have different roles in tumorigenesis and treatment response (36). In our study, PSCs derived from different patient specimens showed different responses to duloxetine. However, we performed experiments with a limited number of PSCs and organoids from one PDAC patient sample. Further study is needed to investigate the response based on the clinical feature and changes in specific fibroblast subtype with duloxetine treatment.

The relationship between antidepressants and cancer has been discussed for over a decade. There is no strong evidence of a relationship between them; however, the relationship between antidepressants and carcinogenesis of colorectal cancer has been reported (37). Other cancers showed a tumor-suppressive effect by exhibiting antidepressant-like properties in a mouse experiment $(38,39)$. Duloxetine has already been used in patients with chronic pains or painful chemotherapy-induced peripheral neuropathy $(40,41)$; therefore, it is well-tolerated for clinical use. Kajiwara et al recently demonstrated that duloxetine treatment improved cancer-associated pain in a PDAC mouse model (42). They stated that duloxetine inhibited the proliferation of PCCs and CAFs, which was consistent with our report. Based on our results, duloxetine is a potential drug for reducing the adverse effects of chemotherapy and enhancing the effect of chemotherapy by targeting stromal remodeling.

\section{Acknowledgements}

The authors would like to thank Ms. Emiko Manabe and Ms. Shoko Sadatomi (Department of Surgery and Oncology, Kyushu University Hospital, Fukuoka, Japan) for their technical assistance during experiments.

\section{Funding}

This study was supported in part by the Japan Society for the Promotion of Science Grants-in-Aid (B) and (C), and a Young Scientists Grant (grant nos. JP18H02880, JP19H03732, JP19K18153 and JP20H03754), the Takeda Science Foundation, Kobayashi Foundation for Cancer Research and The Shinnihon Foundation of Advanced Medical Treatment Research.

\section{Availability of data and materials}

The datasets used and/or analyzed during the current study are available from the corresponding author on reasonable request.

\section{Authors' contributions}

AS, KN and MN conceived and designed the study. AS performed the experiments, data collection and wrote the manuscript. SM designed the experimental approach and contributed to data interpretation and discussion. WG, TS and CI helped with the investigation and formal analysis of data. $\mathrm{NI}$ and KO contributed to the methodology and data analysis. $\mathrm{KN}$ and MN supervised the project, obtained funding and confirm the authenticity of all the raw data. AS and KN revised the manuscript. MN approved the final version of the manuscript. All authors read and approved the final manuscript.

\section{Ethics approval and consent to participate}

Written informed consent was obtained from all participants prior to using their pancreatic cancer surgical specimens for the establishment of pancreatic stellate cells and human organoids. This study was approved by the Ethics Committee of Kyushu University (approval no. IRB: 28-189; Fukuoka, Japan), and all experiments were conducted according to the Ethical Guidelines for Human Genome/Gene Research enacted by the Japanese Government and the Declaration of Helsinki. 


\section{Patient consent for publication}

Not applicable.

\section{Competing interests}

The authors declare that they have no competing interests.

\section{References}

1. Rawla P, Sunkara T and Gaduputi V: Epidemiology of pancreatic cancer: Global trends, etiology and risk factors. World J Oncol 10: 10-27, 2019.

2. Binenbaum Y, Na'ara S and Gil Z: Gemcitabine resistance in pancreatic ductal adenocarcinoma. Drug Resist Updat 23: 55-68, 2015.

3. Parvathaneni V, Kulkarni NS, Muth A and Gupta V: Drug repurposing: A promising tool to accelerate the drug discovery process. Drug Discov Today 24: 2076-2085, 2019.

4. Pushpakom S, Iorio F, Eyers PA, Escott KJ, Hopper S, Wells A Doig A, Guilliams T, Latimer J, McNamee C, et al: Drug repurposing: Progress, challenges and recommendations. Nat Rev Drug Discov 18: 41-58, 2018.

5. Apte MV, Haber PS, Darby SJ, Rodgers SC, McCaughan GW, Korsten MA, Pirola RC and Wilson JS: Pancreatic stellate cells are activated by proinflammatory cytokines: Implications for pancreatic fibrogenesis. Gut 44: 534-541, 1999

6. Apte MV, Wilson JS, Lugea A and Pandol SJ: A starring role for stellate cells in the pancreatic cancer microenvironment. Gastroenterology 144: 1210-1219, 2013.

7. Bachem MG, Schünemann M, Ramadani M, Siech M, Beger H, Buck A, Zhou S, Schmid-Kotsas A and Adler G: Pancreatic carcinoma cells induce fibrosis by stimulating proliferation and matrix synthesis of stellate cells. Gastroenterology 128: 907-921, 2005.

8. Bachem MG, Schneider E, Gross H, Weidenbach H, Schmid RM, Menke A, Siech M, Beger H, Grünert A and Adler G: Identification, culture, and characterization of pancreatic stellate cells in rats and humans. Gastroenterology 115: 421-432, 1998.

9. Boj SF, Hwang CI, Baker LA, Chio II, Engle DD, Corbo V, Jager M, Ponz-Sarvise M, Tiriac H, Spector MS, et al: Organoid models of human and mouse ductal pancreatic cancer. Cell 160: 324-338, 2015.

10. Koikawa K, Ohuchida K, Ando Y, Kibe S, Nakayama H, Takesue S, Endo S, Abe T, Okumura T, Iwamoto C, et al: Basement membrane destruction by pancreatic stellate cells leads to local invasion in pancreatic ductal adenocarcinoma. Cancer Lett 425: 65-77, 2018.

11. Endo S, Nakata K, Ohuchida K, Takesue S, Nakayama H, Abe T, Koikawa K, Okumura T, Sada M, Horioka K, et al: Autophagy is required for activation of pancreatic stellate cells, associated with pancreatic cancer progression and promotes growth of pancreatic tumors in mice. Gastroenterology 152: 1492-1506.e24, 2017.

12. Sagara A, Nakata K, Yamashita T, Guan W, Zhong P, Matsumoto S, Endo S, Iwamoto C, Shindo K, Ikenaga N, et al: New high-throughput screening detects compounds that suppress pancreatic stellate cell activation and attenuate pancreatic cancer growth. Pancreatology: Apr 20, 2021 (Epub ahead of print). doi: 10.1016/j.pan.2021.04.002.

13. Livak KJ and Schmittgen TD: Analysis of relative gene expression data using real-time quantitative PCR and the 2(-Delta Delta C(T)) method. Methods 25: 402-408, 2001.

14. Özdemir BC, Pentcheva-Hoang T, Carstens JL, Zheng X, Wu CC, Simpson TR, Laklai H, Sugimoto H, Kahlert C, Novitskiy SV, et al: Depletion of carcinoma-associated fibroblasts and fibrosis induces immunosuppression and accelerates pancreas cancer with reduced survival. Cancer Cell 25: 719-734, 2014.

15. Xiao Y, Zhang H, Ma Q, Huang R, Lu J, Liang X, Liu X, Zhang Z, Yu L, Pang J, et al: YAP1-mediated pancreatic stellate cell activation inhibits pancreatic cancer cell proliferation. Cancer Lett 462: 51-60, 2019.

16. Jakubowska MA, Ferdek PE, Gerasimenko OV, Gerasimenko JV and Petersen $\mathrm{OH}$ : Nitric oxide signals are interlinked with calcium signals in normal pancreatic stellate cells upon oxidative stress and inflammation. Open Biol 6: 160149, 2016.
17. Jahchan NS, Dudley JT, Mazur PK, Flores N, Yang D, Palmerton A, Zmoos AF, Vaka D, Tran KQT, Zhou M, et al: A drug repositioning approach identifies tricyclic antidepressants as inhibitors of small cell lung cancer and other neuroendocrine tumors. Cancer Discov 3: 1364-1377, 2013.

18. Marescal $\mathrm{O}$ and Cheeseman IM: Cellular mechanisms and regulation of quiescence. Dev Cell 55: 259-271, 2020.

19. Yamaguchi E, Wang C, Fukazawa A, Taki M, Sato Y, Sasaki T, Ueda M, Sasaki N, Higashiyama T and Yamaguchi S: Environment-sensitive fluorescent probe: A benzophosphole oxide with an electron-donating substituent. Angew Chemie Int Ed 54: 4539-4543, 2015.

20. Pampaloni F, Reynaud EG and Stelzer EHK: The third dimension bridges the gap between cell culture and live tissue. Nat Rev Mol Cell Biol 8: 839-845, 2007.

21. Kozono S, Ohuchida K, Eguchi D, Ikenaga N, Fujiwara K, Cui L, Mizumoto K and Tanaka M: Pirfenidone inhibits pancreatic cancer desmoplasia by regulating stellate cells. Cancer Res 73: 2345-2356, 2013

22. Bynigeri RR, Jakkampudi A, Jangala R, Subramanyam C, Sasikala M, Rao GV, Reddy DN and Talukdar R: Pancreatic stellate cell: Pandora's box for pancreatic disease biology. World J Gastroenterol 23: 382-405, 2017.

23. Yu LG, Packman LC, Weldon M, Hamlett J and Rhodes JM: Protein phosphatase $2 \mathrm{~A}$, a negative regulator of the ERK signaling pathway, is activated by tyrosine phosphorylation of putative HLA class II-associated protein I (PHAPI)/pp32 in response to the antiproliferative lectin, jacalin. J Biol Chem 279: 41377-41383, 2004.

24. Janssens V and Goris J: Protein phosphatase 2A: A highly regulated family of serine/threonine phosphatases implicated in cell growth and signalling. Biochem J 353: 417-439, 2001.

25. Naetar N, Soundarapandian V, Litovchick L, Goguen KL, Sablina AA, Bowman-Colin C, Sicinski P, Hahn WC, DeCaprio JA and Livingston DM: PP2A-mediated regulation of ras signaling in $\mathrm{G} 2$ is essential for stable quiescence and normal G1 length. Mol Cell 54: 932-945, 2014.

26. Li L and Hanahan D: Hijacking the neuronal NMDAR signaling circuit to promote tumor growth and invasion. Cell 153: 86-100, 2013.

27. Arakaki AKS, Pan WA and Trejo JA: GPCRs in cancer: Proteaseactivated receptors, endocytic adaptors and signaling. Int $\mathrm{J}$ Mol Sci 19: 1886, 2018.

28. Shiraishi Y, Natsume M, Kofuku Y, Imai S, Nakata K, Mizukoshi T, Ueda T, Iwaï H and Shimada I: Phosphorylationinduced conformation of $\beta_{2}$-adrenoceptor related to arrestin recruitment revealed by NMR. Nat Commun 9: 194, 2018.

29. Nieto Gutierrez A and McDonald PH: GPCRs: Emerging anticancer drug targets. Cell Signal 41: 65-74, 2018.

30. Cortes E, Sarper M, Robinson B, Lachowski D, Chronopoulos A, Thorpe SD, Lee DA and Hernández AE: GPER is a mechanoregulator of pancreatic stellate cells and the tumor microenvironment. EMBO Rep 20: e46556, 2019.

31. Wiley SZ, Sriram K, Liang W, Chang SE, French R, McCann T, Sicklick J, Nishihara H, Lowy AM and Insel PA: GPR68, a proton-sensing GPCR, mediates interaction of cancer-associated fibroblasts and cancer cells. FASEB J 32: 1170-1183, 2018.

32. Lappano R and Maggiolini M: G protein-coupled receptors: Novel targets for drug discovery in cancer. Nat Rev Drug Discov 10: 47-60, 2011.

33. Omary MB, Lugea A, Lowe AW and Pandol SJ: The pancreatic stellate cell: A star on the rise in pancreatic diseases. J Clin Invest 117: 50-59, 2007

34. Miyamoto H, Murakami T, Tsuchida K, Sugino H, Miyake H and Tashiro S: Tumor-stroma interaction of human pancreatic cancer: Acquired resistance to anticancer drugs and proliferation regulation is dependent on extracellular matrix proteins. Pancreas 28: $38-44,2004$.

35. Erkan M,Kleeff J, Gorbachevski A, Reiser C,Mitkus T, Esposito I, Giese T, Büchler MW, Giese NA and Friess H: Periostin creates a tumor-supportive microenvironment in the pancreas by sustaining fibrogenic stellate cell activity. Gastroenterology 132: 1447-1464, 2007

36. Öhlund D, Handly-Santana A, Biffi G, Elyada E, Almeida AS, Ponz-Sarvise M, Corbo V, Oni TE, Hearn SA, Lee EJ, et al: Distinct populations of inflammatory fibroblasts and myofibroblasts in pancreatic cancer. J Exp Med 214: 579-596, 2017.

37. Xu W, Tamim H, Shapiro S, Stang MR and Collet JP: Use of antidepressants and risk of colorectal cancer: A nested case-control study. Lancet Oncol 7: 301-308, 2006. 
38. Tegowski M, Fan $\mathrm{C}$ and Baldwin AS: Thioridazine inhibits self-renewal in breast cancer cells via DRD2-dependent STAT3 inhibition, but induces a G1 arrest independent of DRD2. J Biol Chem 293: 15977-15990, 2018.

39. Gwynne WD, Hallett RM, Girgis-Gabardo A, Bojovic B, Dvorkin-Gheva A, Aarts C, Dias K, Bane A and Hassell JA: Serotonergic system antagonists target breast tumor initiating cells and synergize with chemotherapy to shrink human breast tumor xenografts. Oncotarget 8: 32101-32116, 2017.

40. Smith EML, Pang H, Cirrincione C, Fleishman S, Paskett ED, Ahles T, Bressler LR, Fadul CE, Knox C, Le-Lindqwister N, et al: Effect of duloxetine on pain, function, and quality of life among patients with chemotherapy-induced painful peripheral neuropathy: A randomized clinical trial. JAMA 309: 1359-1367, 2013.
41. Hirayama Y, Ishitani K, Sato Y, Iyama S, Takada K, Murase K, Kuroda H, Nagamachi Y, Konuma Y, Fujimi A, et al: Effect of duloxetine in Japanese patients with chemotherapy-induced peripheral neuropathy: A pilot randomized trial. Int J Clin Oncol 20: 866-871, 2015.

42. Kajiwara I, Sano M, Ichimaru Y, Oshima Y, Kitajima O, Hao H, Masamune A, Kim J, Ishii Y, Ijichi H, et al: Duloxetine improves cancer-associated pain in a mouse model of pancreatic cancer through stimulation of noradrenaline pathway and its antitumor effects. Pain 161: 2909-2919, 2020.

This work is licensed under a Creative Commons Attribution-NonCommercial-NoDerivatives 4.0 International (CC BY-NC-ND 4.0) License. 